\title{
Low Dimensional Carbon Materials for Applications in Mass and Energy Transport
}

\author{
Qing Hua Wang, ${ }^{\dagger}$ Darin O. Bellisario, ${ }^{\ddagger}$ Lee W. Drahushuk, ${ }^{\dagger}$ Rishabh M. Jain, ${ }^{\S}$ Sebastian Kruss, ${ }^{\dagger}$

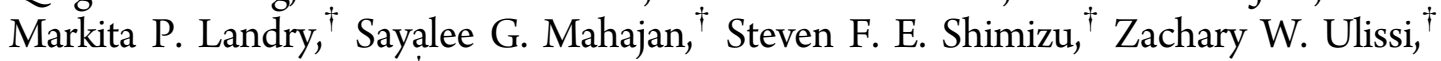 \\ and Michael S. Strano* ${ }^{* \dagger}$ \\ ${ }^{\dagger}$ Department of Chemical Engineering, Massachusetts Institute of Technology, Cambridge, Massachusetts 02139, United States \\ ${ }^{\ddagger}$ Department of Chemistry, Massachusetts Institute of Technology, Cambridge, Massachusetts 02139, United States \\ ${ }^{\S}$ Department of Materials Science and Engineering, Massachusetts Institute of Technology, Cambridge, Massachusetts 02139, United \\ States
}

ABSTRACT: Low dimensional materials are those that possess at least one physical boundary small enough to confine the electrons or phonons. This quantum confinement reduces the dimensionality of the material and imparts unique and novel properties that are not seen in their bulk forms. Examples include quantum dots (0-D), carbon nanotubes (1-D), and graphene (2-D). Accordingly, these materials exhibit new concepts in mass and energy transport that

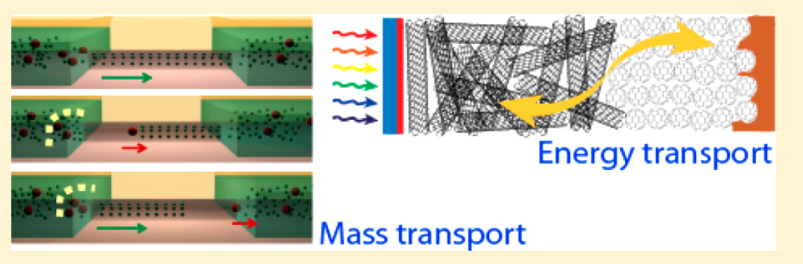
can be exploited for technological applications. In this Perspective, we review several topics related to mass and energy transport in and around carbon-based low dimensional materials. Recent developments in the study of matter being transported through carbon nanotube and graphene nanopores are reviewed, as well as applications of excitonic, thermal, and electronic energy transport in carbon nanotubes. The nanometer-scale interior of a single-walled carbon nanotube (SWCNT) has been studied as a unique nanopore, exhibiting periodic ionic conduction currents and dimensionally confined material phases. The mechanism of gas transport through atomic-scale holes in graphene, which is otherwise a perfect barrier material, has been analytically studied. These insights on nanoscale mass transport will have important implications in systems ranging from biological nanopores to advanced water filtration devices. The electronic structure of semiconducting SWCNTs allows photogenerated excitons to be harnessed for single-molecule biosensing and as elements of a new class of all-nanocarbon near-infrared photovoltaics. The extremely high thermal and electrical conductivities of carbon nanotubes allows the generation of electrical energy from chemical reactions. The understanding of how low dimensional physics and chemistry influences mass and energy transport will facilitate the application of these materials to a variety of scientific challenges.

KEYWORDS: carbon nanotubes, graphene, nanopores, excitons, photovoltaics, biosensing, thermopower

\section{INTRODUCTION}

Low dimensional materials composed of carbon, carbon nanomaterials, are a remarkable class of materials with unique properties. Graphene is a single atomic sheet of carbon in a hexagonal lattice. Because of its band structure, its carriers behave as massless Dirac fermions, resulting in exceptionally high mobilities. It is also extremely strong because of its $\mathrm{sp}^{2}$ bonded carbon lattice. Rolling a sheet of graphene into a seamless cylinder results in a single-walled carbon nanotube (SWCNT). Depending on the direction of rolling, or the chiral vector $(n, m)$, the resulting SWCNT can be semiconducting or metallic. CNTs share the mechanical strength of graphene, but the electronic structure has many features not seen in graphene, such as the van Hove singularities arising from the onedimensional confinement, that lead to many interesting optical and electronic effects. While the electronic, mechanical, optical, and chemical properties and applications of both CNTs and graphene have been widely studied with many remarkable results, in this Perspective, we focus on efforts in our lab and others to study the unique features of carbon nanomaterials in mass and energy transport and to understand and exploit them in several types of applications. In the first section, we review the transport of mass, in particular, liquids, gases, and ions, through carbon nanopores such as the interiors of SWCNTs and nanometer-scale holes in graphene sheets. In the second section, we review three applications of energy transfer in CNTs: in fluorescent near-infrared (nIR) biosensing, in photovoltaics, and in converting chemical to electrical energy in thermopower waves. We conclude with some perspectives on future directions for mass and energy transport in carbon nanomaterials and other nanomaterials.

Special Issue: Celebrating Twenty-Five Years of Chemistry of Materials

Received: August 28, 2013

Revised: October 7, 2013

Published: October 21, 2013 


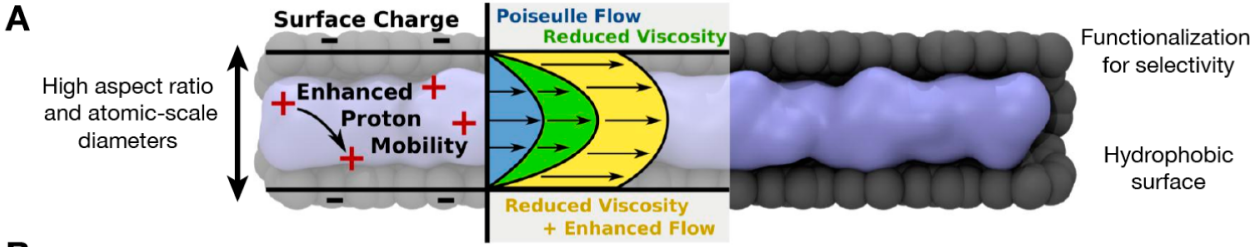

B

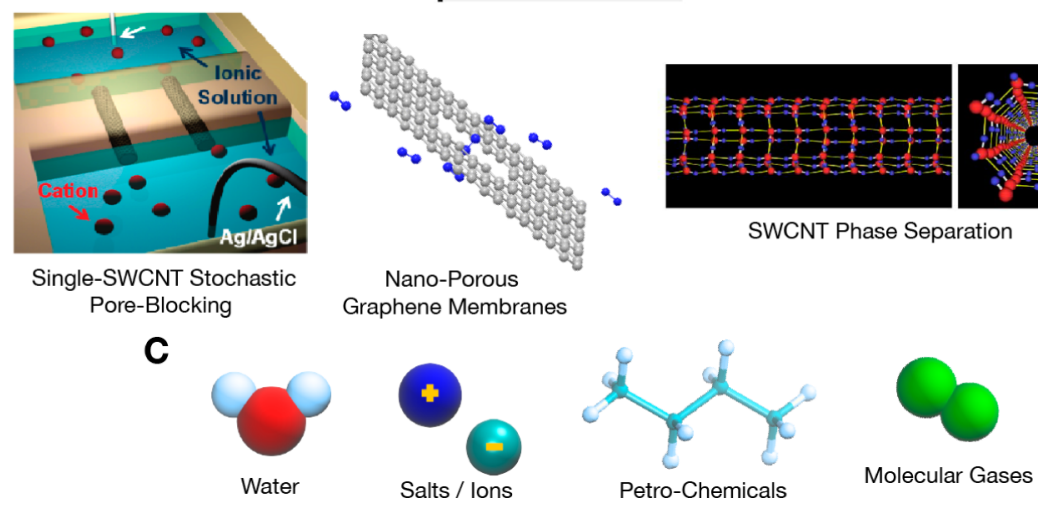

Figure 1. Overview of molecular transport through carbon nanomaterial nanopores. (A) Features of nanocarbon materials that contribute to interesting transport properties. Adapted with permission from ref 11. Copyright 2013 Nature Publishing Group. (B) Three types of devices and systems for studying molecular transport through carbon nanotubes (Adapted from ref 6. Copyright 2010 American Chemical Society.), graphene membranes with defined nanopores (Adapted from ref 12. Copyright 2012 American Chemical Society.), and phase separation inside SWCNTs (Adapted with permission from ref 7. Copyright 2008 National Academy of Sciences, USA.). (C) Some of the molecules exhibiting interesting transport behavior with carbon nanopores.

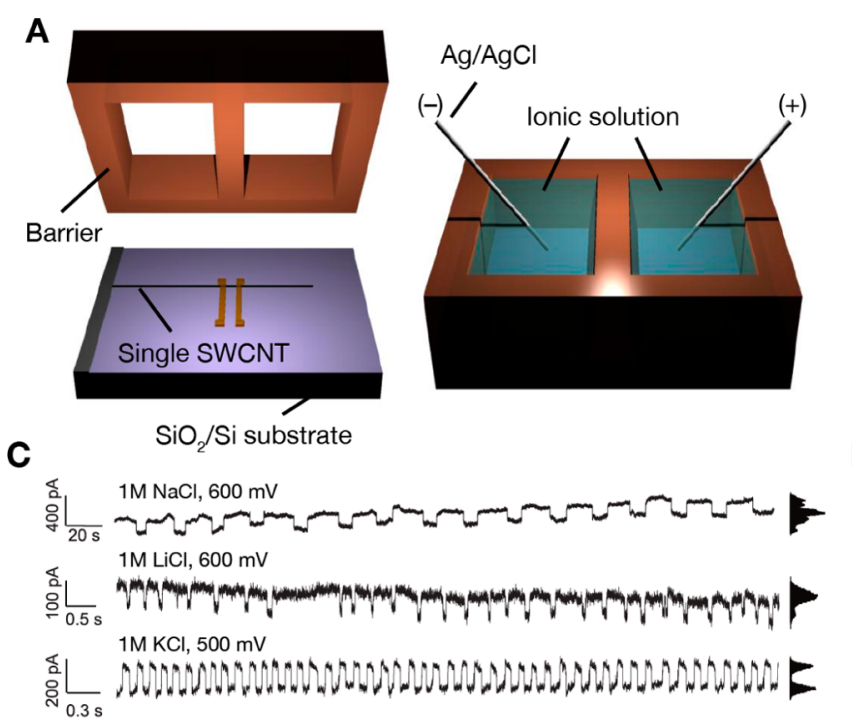

B

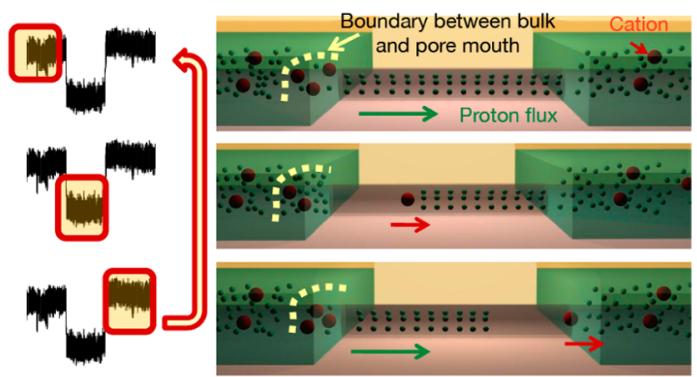

D 500

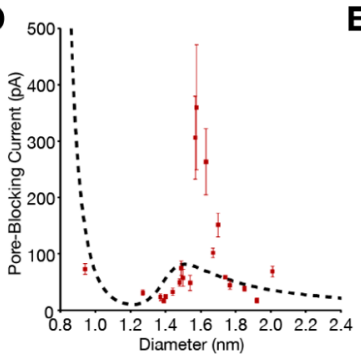

E

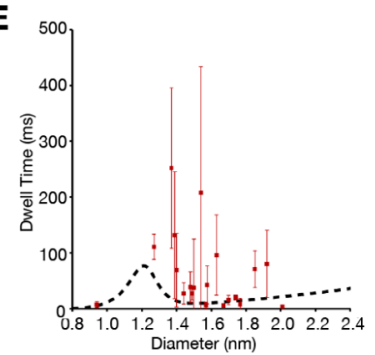

Figure 2. Ion transport through individual SWCNT nanopores. (A) Typical device consisting of aligned SWCNT grown on silicon dioxide cross a barrier between two reservoirs. ${ }^{11}$ (B) Cartoon of a pore-blocking event, where an accelerated proton flux is reduced by the presence of a larger cation. ${ }^{11}(\mathrm{C})$ Three examples of coherence resonance in SWCNT devices, with sustained regular oscillations. ${ }^{5}$ (D, E) Diameter dependence of poreblocking current and dwell times, showing a maximum in pore-blocking current for diameters of approximately $1.6 \mathrm{~nm} .{ }^{11}$ Panels A, B, D, and E adapted with permission from ref 11. Copyright 2013 Nature Publishing Group. Panel C adapted with permission from ref 5. Copyright 2010 American Association for the Advancement of Science.

\section{MOLECULAR TRANSPORT}

2.1. Introduction to Molecular Transport through Carbon Nanotubes and Graphene. Carbon nanomaterials such as carbon nanotubes and graphene have been studied extensively for their exceptional mechanical, electrical, and optical properties. Recently, however, there has been increased interest in studying their molecular transport, phase behavior, and barrier membrane properties. ${ }^{1-4}$ Several properties of nanopores based on CNTs and graphene make them well suited for studying molecular transport and filling. They have molecular-scale dimensions (typical diameters 0.8 to $2 \mathrm{~nm}$ ) which can achieve size-based partitioning of molecules, ${ }^{1}$ make it suitable for single-molecule detection platforms, ${ }^{5,6}$ and can significantly change phase behavior in confined fluids. ${ }^{7}$ The pores' hydrophobic nature and atomically smooth surface create slip at the wall boundaries, thus enhancing rates of 
transport above that predicted by Poiseuille flow by orders of magnitude. ${ }^{1,8}$ Furthermore, the length of these pores can range from a single atom layer thick for graphene, ${ }^{2}$ to millimeters in length for straight-line carbon nanotube nanopores. ${ }^{5}$ Very low aspect ratios can increase the efficiency of membrane filtration, while long aspect ratios can be advantageous for singlemolecule detection. ${ }^{5}$ In addition, the carbon-based structure allows attachment of a wide range of functional groups that can modify the partitioning of different molecules inside the pore. ${ }^{9,10}$ These concepts are summarized in Figure 1. A greater understanding of nanopores has many uses, for example, in the study of biological nanopores, DNA sequencing, gas filtration, water desalination, and molecular detection.

In this section, we review recent work conducted in our group and from the literature on the experimental and theoretical transport of molecules through nanoscale pores in carbon nanomaterials. We have studied the transport of ions through individual single-walled carbon nanotubes (SWCNTs), different phases of matter inside nanopores, and the mechanism of gas transport through graphene nanopores.

2.2. Carbon Nanotube Nanopores as Molecular Conduits. Single-walled carbon nanotubes (SWCNTs) provide an excellent platform for the study of molecular transport in nanoscale environments. They have very small diameters (usually less than $2 \mathrm{~nm}$ ), that can be experimentally identified, and smooth hydrophobic interiors that lend themselves to the study of molecular transport at the nanoscale. Understanding transport in these devices is broadly applicable to studying similar biological ion channels and to designing new membranes and devices for separation processes and desalination.

A typical two-reservoir SWCNT device, as used in a variety of studies, $5,6,11,13,14$ is shown in Figure 1A. Aligned SWCNTs are first grown on a silicon dioxide substrate and a two-reservoir barrier system built on top. Different studies have customized this setup by studying transport through devices with two SWCNTs, ${ }^{6}$ characterizing each SWCNT, ${ }^{11}$ or altering the electronic structure with an applied gate voltage. ${ }^{13,14}$ These devices are unique in the electrochemical literature in that they allow the study of transport through single nanopores with controllable diameters of less than $2 \mathrm{~nm}$.

Using the experimental platform described above, we found that the transport of water and ions in confined regions of molecular scale leads to stochastic current fluctuations, as shown in Figure 2B. ${ }^{5}$ Accelerated proton and water transport leads to high current transport rates. This proton current can be disrupted upon the introduction of cations, with the type of cation affecting the current drop (pore-blocking current) and length scale (dwell time). This effect is similar to the stochastic current fluctuations in biological ion channels with similar nanometer-scale diameters. These stochastic fluctuations can also be observed in systems with a small number of SWCNTs. ${ }^{6}$ In a subset of these devices, ionic blocking can lead to a selfreinforcing oscillation leading to highly regular current fluctuations known as coherence resonance 5 and shown in Figure 2C for several types of ions. These sustained oscillations are unique for nanopores with atomic-scale radii.

We have recently developed device structures to measure ion transport properties through individual well-characterized SWCNTs, ${ }^{11}$ illustrated in Figure 2A. Each SWCNT was characterized with Raman spectroscopy allowing for an assignment of the diameter and the electronic type (semiconducting or metallic). Using a large number of devices with
SWCNTs of varying diameters, we investigated the diameter dependence of the observed pore-blocking phenomena, as shown in Figure 2D,E, where a continuum model is indicated by the dashed line. We found a surprising maximum in ion transport rates for SWCNTs with diameters of $\sim 1.6 \mathrm{~nm}$. At small diameters $(<1 \mathrm{~nm})$, the strong internal water structuring dominates and results in greatly enhanced water and proton transport rates. As the diameter increases, there is a transition to accelerated bulk flow at approximately $1.6 \mathrm{~nm}$, as predicted by molecular simulations. ${ }^{15}$ As the diameter increases further, water transport approaches bulk transport rates. This accelerated fluid flow results in enhanced ionic current as observed in a peak in the pore-blocking current at just under $1.6 \mathrm{~nm}$.

The electronic properties of SWCNTs are also important in modulating ionic transport rates. Several studies have investigated transport through single-SWCNT devices with an applied electric field across the SWCNT. ${ }^{13,14}$ Although these devices were not diameter-assigned, we suspect that they had diameters larger than those studied recently in our laboratory ${ }^{5,6,11}$ due to their nonstochastic transport characteristics and large $\mathrm{Ru}(\mathrm{bpy})_{3}{ }^{2+}$ flux rates. Changing the applied voltage drastically changed the observed ionic transport rates. This effect could be particularly useful for electronically modulating the ionic current through SWCNT devices.

There are several possibilities for the further exploration of ionic transport through SWCNTs. Integrating electronic gating to diameter-characterized SWCNT devices would further reveal the precise mechanisms behind accelerated interior transport. Altering the entrance and exit environment of the SWCNT through covalent end-functionalization could alter the selectivity of transport through the interiors by controlling the species that can enter the pore.

2.3. Phase Behavior in Nanopores. Mass transport through the interior of a nanopore is governed by the thermodynamic phase behavior of the fluid in the pore. We typically think about phase transitions as following the Gibbs' phase rule, where the number of intensive variables required to specify the system is equal to $F=C-P_{n}+2$, where $C$ is the number of components and $P_{\mathrm{n}}$ is the number of phases. For example, pure water $(C=1)$ at vapor-liquid equilibrium $\left(P_{\mathrm{n}}=\right.$ 2) and a temperature of $100{ }^{\circ} \mathrm{C}$ completely specifies the system and constrains all the other intensive variables (e.g., pressure must be 1 atm). However, this rule is only valid for bulk systems and does not work in confined nanoscale systems. In a nanopore, the state of the pore fluid also depends strongly on the identity, density, and configuration of atoms making up the pore walls and the surface curvature and geometry of the pore. These factors all modify the free energy of the material inside the pore, and these additional degrees of freedom make studying nanopore phase behavior much more difficult. The differences in bulk and nanoconfined systems are shown in Figure 3A.

While interesting from a fundamental science point of view, there are important reasons for understanding the phase behavior inside of nanopores, especially those of carbon nanotubes. One potential application in the biomedical field is the storage and release of encapsulated molecules from carbon nanotubes, for example, for drug delivery. ${ }^{16}$ The conditions required to store and release molecules from a confined space can be obtained from a modified phase diagram, which would help to screen different molecules and accelerate development of novel release schemes. 

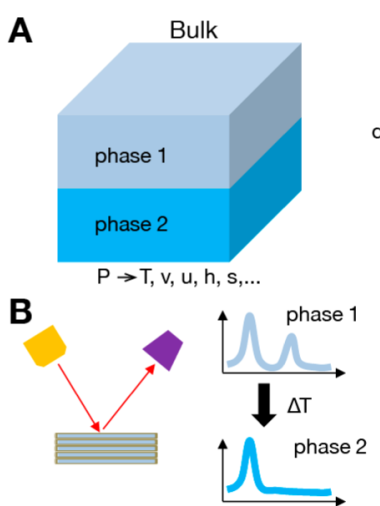

XRD, NMR, Raman, DSC, TGA

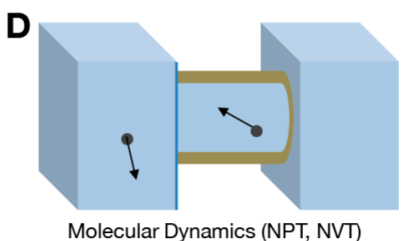

Molecular Dynamics (NPT, NVT)

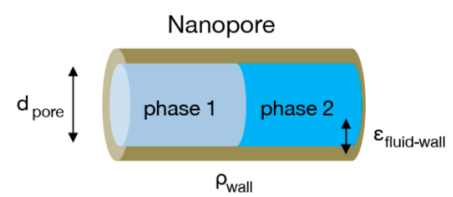

$P, d_{\text {pore }}, P_{\text {wall }}, \varepsilon_{\text {fluid-wall }}, \ldots \rightarrow T, v, u, h, s, \ldots$

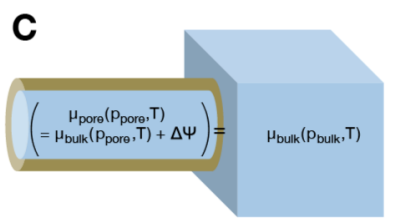

$\Delta \Psi=f\left(d_{\text {pore }}, \rho_{\text {wall }}, \varepsilon_{\text {fluid -wall }}, \ldots\right)$

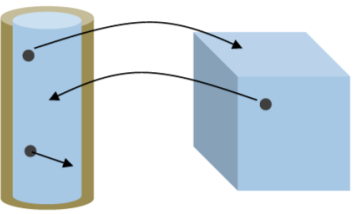

Grand Canonical Monte Carlo $(\mu \mathrm{VT})$

Figure 3. Overview of nanopore phase behavior. (A) For a bulk single component with two phases in coexistence, a single intensive variable specifies the other intensive variables of the system. For example, pressure $(P)$ determines the temperature $(T)$, molar volume $(v)$, molar internal energy $(u)$, molar enthalpy $(h)$, molar entropy $(s)$, etc. However, for a substance confined to a nanopore, there are several other pore properties required to determine the conditions of the phase transition, including the pore diameter $\left(d_{\text {pore }}\right)$, a parameter describing the interaction energy between the fluid and pore walls $\left(\varepsilon_{\text {fluid-wall }}\right)$, and the density of atoms in the pore wall $\left(\rho_{\text {wall }}\right)$. (B) Most experimental methods probe phase transitions in bulk quantities of nanoporous materials by monitoring changes in spectrum, specific heat, or mass as temperature is varied. (C) A few analytical methods have been proposed to predict global phase diagrams, one of which modifies the pore chemical potential $(\mu)$ by some excess potential $(\Delta \Psi)$ induced by the pore confinement. (D) Common simulation methods for studying phase behavior are molecular dynamics and grand canonical Monte Carlo simulations.

A second application of interest is in the use of carbon nanotube nanopores as model systems to explore phase behavior in geological nanopores for oil and gas recovery. ${ }^{17}$ Although increasingly less permeable rocks (e.g., tight sandstones and shale) are explored for oil and gas, there is a lack of understanding about how much hydrocarbons are stored in these nanopores and what conditions are required to displace the hydrocarbons. Permeability, while not an equilibrium quantity, is also required input for standard reservoir models and is not well predicted by conventional permeability models.

A final application may be the potential to carry out reactions inside carbon nanotube frameworks. The confinement effects could modify the free energy of the reactants, transition state, and products differently, thus affecting the reaction equilibrium constant and kinetics. While relatively little explored, it is possible that valuable reactions not favorable in the bulk can occur efficiently inside carbon nanotubes.

Experimental Studies. Several experimental methods have been developed for studying nanopore phase behavior or filling state characterization, as shown in Figure $3 \mathrm{~B}$. Neutron and $\mathrm{X}$ ray diffraction (XRD), techniques that can detect the presence of long-range ordering, have been carried out at a range of temperatures to determine the filling state and the presence of a solid phase inside a bulk collection of nanotubes. ${ }^{18,19}$ Nuclear

magnetic resonance (NMR) spectroscopy is also a unique tool for monitoring the hydrogen atom relaxation times, which vary depending on whether the water, for example, is mobile or solid. ${ }^{20,21}$ Raman spectroscopy has also been employed to study phase transitions in nanopores by probing the change in vibrational modes of the confined fluid. ${ }^{22}$ Finally, thermoanalytical techniques such as differential scanning calorimetry (DSC) and thermogravimetric analysis (TGA) can monitor bulk properties (specific heat and mass, respectively) to indicate phase transitions in bulk nanopore samples. ${ }^{23}$

Analytical Methods. There have been several attempts to theoretically model global phase diagrams of simple fluids confined to cylindrical or slit-shaped pore geometries. Radhakrishnan et al. used the theory of corresponding states to demonstrate that the global phase diagram for nanoconfined Lennard-Jones ( $\mathrm{LJ}$ ) fluids could be produced using primarily the reduced pore width (the pore diameter normalized by the LJ parameter for the fluid) and a parameter indicating the fluid-wall interaction strengths. ${ }^{24}$ Miyahara and co-workers have also developed equations describing the global phase diagram for LJ fluids by modifying the free energy of the confined fluid by an excess interaction energy due to the pore walls compared to the material in the bulk..$^{25-27}$ Future goals will be to extend the predictions of these models to dipolar molecules and larger molecules not well described by the Lennard-Jones model.

Simulation Methods. The most common molecular simulations performed are molecular dynamics (MD) simulations and Monte Carlo simulations. MD simulations for studying phase transitions are usually carried out under constant NPT or NVT conditions (where $\mathrm{N}$ is the number of molecules, $\mathrm{P}$ is thepressure, $\mathrm{V}$ is the volume, and $\mathrm{T}$ the temperature). Multiple simulations are run (each at constant NPT or NVT) while varying the temperatures between simulations until there is a threshold change in the structure factor of the fluid inside the pore or discontinuity in the density. ${ }^{7}$ Numerous MD simulations, especially of water in carbon nanotubes, ${ }^{28}$ have been carried out showing several different phases of water being formed inside the nanotube, usually under constant NVT or NPT conditions.

Monte Carlo simulations are well suited for probing phase transitions and adsorption inside nanopores. In these simulations, typically carried out in the grand canonical ensemble (constant $\mu \mathrm{VT}$ conditions, where $\mu$ is the chemical potential), the pore is initially populated with molecules, and each Monte Carlo move attempts to displace a particle and exchange a particle with the bulk reservoir at constant $\mu$. Thus, these simulations have the advantage in that there are no interfaces between the bulk and the pore which can distort calculation of phase properties and require much larger systems, and the simulations converge to equilibrium faster than MD simulations. ${ }^{29}$ Running the simulations at different temperatures and/or pressures (and using an equation of state to calculate the corresponding chemical potential) while monitoring the density or structure factor of the fluid inside the pore, one can locate the phase transition. ${ }^{30}$ These simulation methods are summarized in Figure 3D.

Comparing Simulations, Theory, and Experiments. Comparisons between simulations, theories, and experiments should be considered with appropriate skepticism. For example, most simulations of fluids inside carbon nanotubes use force field parameters that have been parametrized to recreate various bulk properties (e.g., density, bulk heats of vaporization, radial 

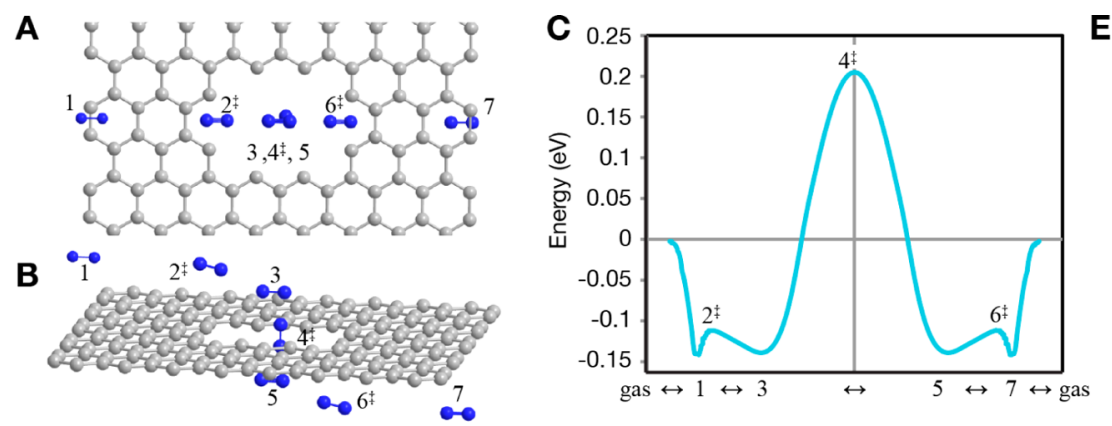

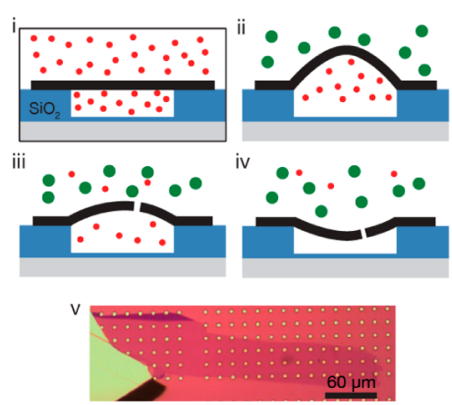

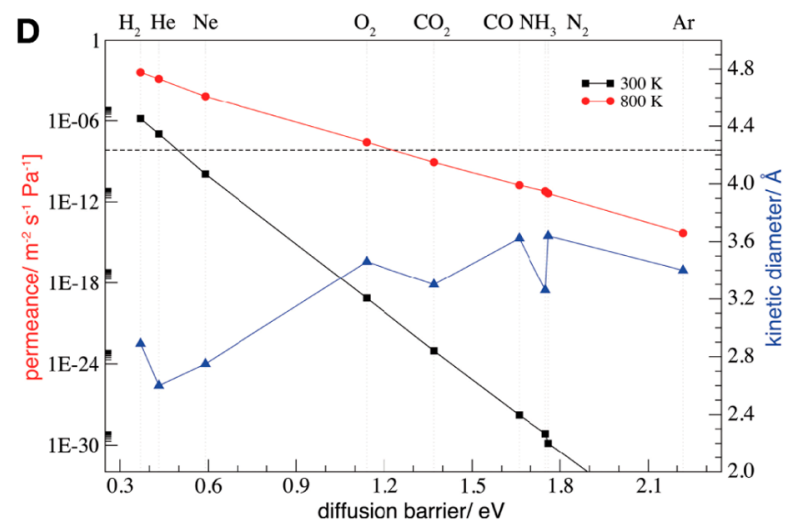

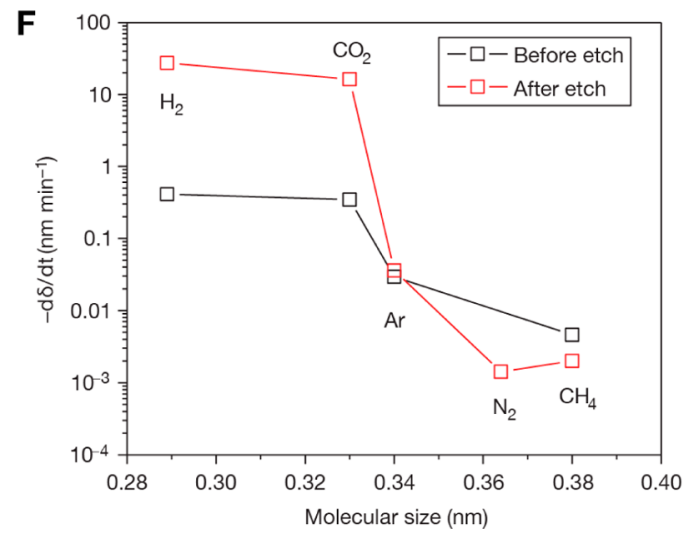

Figure 4. (A) Top view and (B) side view for nitrogen passing through a model decavacancy pore, without functionalization, in single layer graphene along the minimum energy adsorption mechanism. (C) Energy coordinate graph for nitrogen passing through a pore as depicted (A) and (B). The height of the energy barrier at the center is also the same for molecules passing through via the gas phase mechanism. Panels A-C adapted from ref 12. Copyright 2012 American Chemical Society. (D) Calculated energy barriers for nine gases (horizontal axis), plotted along with permeance (left vertical axis) calculated from the gas phase impingement model and with the gas molecules kinetic diameters (right vertical axis) for a hexavacancy pore functionalized with hydrogen. Includes permeance calculated for two temperatures, $300 \mathrm{~K}$ and $800 \mathrm{~K}$, and a horizontal dotted line representing an approximate threshold for commercially viable permeances. Reproduced with permission from ref 37. Copyright 2010 Wiley. (E) (i-iv) Schematic diagrams showing graphene covering a microcavity in $\mathrm{SiO}_{2}$ being pressurized with hydrogen (red dots); chamber expands when in air (green dots); after etching small pores, the hydrogen atoms escape while the air molecules do not enter. (v) Optical image showing sheet of graphene covering many microcavities in $\mathrm{SiO}_{2}$. (F) The molecular effusion rate calculated from experimental measurements with an AFM of the deflection of the bubble over a graphene well, before and after a single pore is etched in the single layer graphene membrane. As only the smaller molecules are able to pass through the manufactured pore, this experiment demonstrates molecular sieving based on size. Panels $\mathrm{E}$ and $\mathrm{F}$ adapted with permission from ref 4. Copyright 2012 Nature Publishing Group.

distribution function, etc.) and are not guaranteed to reproduce accurately behavior in nanoconfined fluids, which is experimentally unknown for the most part. It has been shown, for instance, that small changes $\left(<0.1 k_{\mathrm{B}} T\right.$, where $k_{\mathrm{B}}$ is the Boltzmann constant) in the carbon-water van der Waals potential can result in either emptying or filling of the tube. ${ }^{31}$ $\mathrm{Ab}$ initio calculations could be carried out which require less parametrization, but they are also much more computationally expensive and limited to studying tens to hundreds of molecules. $^{32}$

Furthermore, most simulations ignore the long-range polarizability of carbon nanotubes which may be either very significant or negligible depending on the polarity of the molecule, the metallicity and length of the tube, and the presence of other molecules in the system. ${ }^{33,34}$ It is also important to simulate experimentally relevant geometry and conditions, such as carbon nanotubes on a silicon dioxide substrate or as part of a bundle and with the appropriate endgroup functionalization (typically, oxidation of the carbon nanotube ends results in carboxylic acid groups attached on the ends).

2.4. Graphene as a Membrane for Gas Transport. A novel application of graphene is to act as an atomically thin separation membrane. It has been shown that a single layer of pristine graphene is impermeable to gases. ${ }^{35}$ Furthermore, theoretical calculations of graphene containing nanoscale pores have shown that such membranes are capable of highly selective separations. ${ }^{36,37}$ Recent experimental work has shown selectivity with gases ${ }^{4}$ and aqueous solutes. ${ }^{38}$ As a $2 \mathrm{D}$ atomically thin material, graphene's properties give it new advantages over typical membrane materials, whose characteristics are determined by the bulk properties throughout the thickness of the membrane.

For a typical bulk separation membrane, there is a trade-off between the permeance and selectivity, and the thickness of the membrane determines the balance between the two. For a single layer graphene membrane, there is effectively no thickness and both its permeance and selectivity are typically dominated by a single molecular energy barrier for crossing through a manufactured pore in the graphene. High separation factors and high permeances are simultaneously possible in precisely defined nanopores in the atomic lattice of carbon in graphene. The basic mechanism by which gas molecules can permeate through the pore is gas phase impingement on the pore area with high enough kinetic energy to overcome the energy barrier for passing through. Density functional theory (DFT) has been used to calculate the energetics and predict separation factors for the separation of hydrogen and methane 
with a variety of pore configurations. ${ }^{36}$ These results demonstrated that the pore configuration could be tuned such that the energy barrier for methane to pass was much higher than for hydrogen, which resulted in predicted separation factors of $10^{8}$ and $10^{23}$ for different pores. Other work examined the energy barriers for nine different gases, ranging in size from hydrogen to argon, passing through a single type of pore. ${ }^{37}$ Those results also predicted high selectivities and showed that there were relatively large differences in the energy barrier correlating loosely with kinetic diameter, as shown in Figure 4D.

Another effect of the atomic thickness is that adsorption to the external surface of the graphene can play a significant role in the permeation of gases, in addition to the standard gas phase impingement mechanism. Molecular dynamics (MD) simulations have shown an increased probability for molecules to be located near the surface, indicating absorption is taking place $^{39,40}$ Once they are adsorbed to the surface, molecules can move to the pore and pass through. A simple description of adsorption-mediated transport is a permeation rate that is proportional to the surface coverage from the Langmuir adsorption model. ${ }^{40}$ In one of the MD simulations, it was observed that the permeation of nitrogen leveled off and remained constant as the size of the pore was increased, but the permeation of hydrogen continued to increase approximately linearly. ${ }^{39}$ This is an example of phenomenon that cannot be explained only with the simple gas phase impingement model.

Relying on concepts from surface science and heterogeneous catalysis, a more inclusive model can be used to describe the apparent insensitivity to pore size. ${ }^{12}$ This model uses multiple steps describing the adsorption and desorption, entering and leaving the pore area, and passing through the pore. An example of this process with the molecular positions and corresponding energy is shown in Figure 4A-C. Different analytical expressions can be used to describe the permeation depending on which of those processes are dominant. The insensitivity of the nitrogen permeation rate to pore size in the $\mathrm{MD}$ simulation can be explained as a result of the energy barrier to approach the pore, which is the rate limiting step and approximately independent of the pore size. The molecular dynamics of hydrogen does not show this behavior because it adsorbs to the graphene surface less and the overall permeation rate is still dominated by the gas phase impingement mechanism. These results show there are multiple interesting mechanisms that are present and can be manipulated to control permeation in single layer graphene membranes.

Experiments have also demonstrated the high separation factors indicative of molecular sieving at the graphene pore. In the work of Koenig et al., ${ }^{4}$ mechanically exfoliated graphene was suspended over a well etched in silicon dioxide, as shown in Figure 4E,F. The well underneath the single layer graphene flake was charged with gas by leaving it in a pressure chamber long enough for diffusion through the silicon oxide to fully pressurize the internal chamber. By carefully etching with ozone while monitoring for a sudden increase in the permeation rate, a single pore is made in the region of the well. It is assumed this pore is functionalized with oxygen moieties; however, no direct characterization has been done to confirm this, and the exact atomic structure and chemical nature of the pore is an open question. Pore functionalization is expected to play a role in determining selectivity and could be used to modulate the characteristics of separation, and more investigation is required in this area.
After the pore has been formed, the well can be again charged with a gas and the pressure, and therefore quantity, of gas within the well can be monitored by using an atomic force microscope (AFM) to measure the height of the graphene bubble that forms from the pressure within the well. By monitoring the rate of change over time, the permeation rates for various gases were measured through the single pore. Over the gases tested $\left(\mathrm{H}_{2}, \mathrm{CO}_{2}, \mathrm{Ar}, \mathrm{N}_{2}, \mathrm{CH}_{4}, \mathrm{SF}_{6}\right)$, the permeation rate was high for hydrogen and carbon dioxide but dropped off at higher molecular size and became commensurate with the leak rate observed before the pore was created, which was orders of magnitude lower than the rate for hydrogen and carbon dioxide. The permeation rate for the small gases was also consistent with the order of magnitude predicted by the impingement rate from the kinetic theory of gases. These results are shown in Figure 4F. This validates the predicted high selectivity and molecular sieving that can be achieved with single layer graphene membranes. We also note that the molecules being differentiated are very close in size, showing the effect of the atomic-scale pores.

The previous experimental demonstration of molecular sieving was performed on a small-scale system. Practical applications of graphene membranes will involve larger areas, such as the work of O'Hern et al. ${ }^{38}$ that uses graphene grown by chemical vapor deposition (CVD) with an aqueous system. The simulations, models, and early experiment work all demonstrate the vast potential of single layer graphene membranes for separations.

\section{ENERGY TRANSPORT}

\subsection{Introduction to Energy Transport in Carbon} Nanomaterials. For applications in energy transfer, the electronic structure and phonon structure of CNTs, and sometimes the interaction between them, are very important. In particular, semiconducting SWCNTs exhibit photoluminescence (PL) due to the absorption of light to generate excitons, whose energy can then be released as light. In optoelectronic applications, the low dimensional confinement in SWCNTs is very important. The limited high wavelength phonon modes minimize exciton and charge scattering in carbon nanotubes, leading to mobilities and diffusivities orders of magnitude greater than traditional d-orbital metals and semiconductors. This property has made SWCNT attractive for photovoltaics in particular, both as a scaffold for conveying carriers ${ }^{41,42}$ and, combined with its high absorption cross-section (on the order of $10^{-17} \mathrm{~cm}^{2}$ per carbon atom ${ }^{43}$ ), for primary absorption. Recently, the low cost possibilities presented by an all-carbon photovoltaic based on SWCNT and fullerenes, which can be produced by scalable methods such as chemical vapor deposition (CVD) and solution phase processing, have been explored with great success. ${ }^{41,44}$ The phonon modes in SWCNTs are also highly anisotropic due to the 1D confinement, such that the thermal conductivity along the axis is almost 10 times higher than that of copper. ${ }^{45}$ The thermal conductivity can be harnessed to produce selfpropagating thermal waves from exothermically reacting fuels, and coupling the phonon modes to electrons generates an electrical pulse. ${ }^{17,18}$

In this section, we review recent results from our lab and in the literature on three diverse aspects of energy transport in carbon nanotubes. We have explored SWCNTs as fluorescent optical sensors for single molecule detection, ${ }^{46-48}$ where photogenerated excitons are influenced by the presence of 

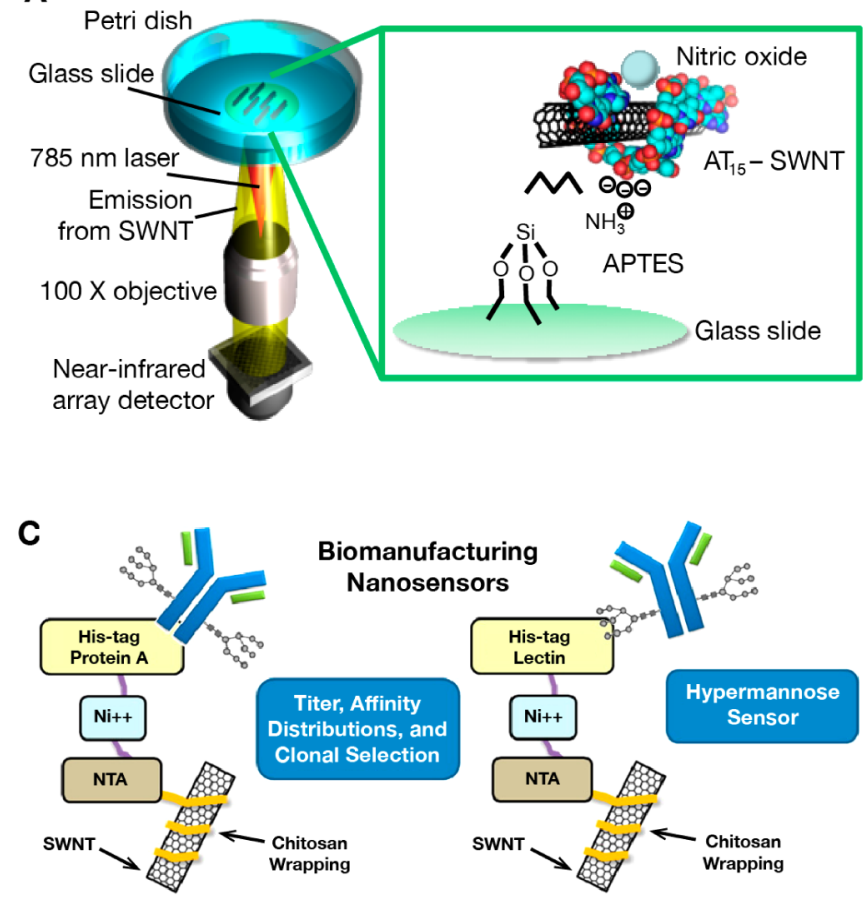

B

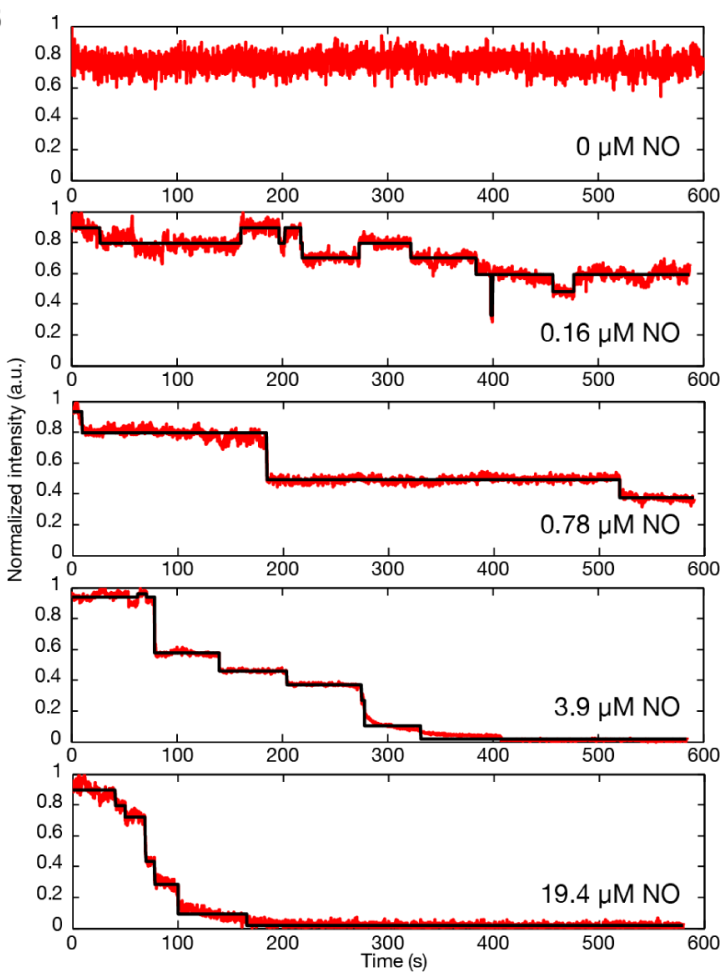

Figure 5. SWCNT-based biosensors. (A) Schematic for fluorescent detection of NO by (AT) 15 -DNA wrapped SWCNTs. (B) Normalized fluorescence intensity traces for single SWCNT upon exposure to increasing concentrations of NO. When a NO molecule adsorbs to the sensor, discrete quenching steps occur which can be used to determine NO concentrations and rate constants. (C) Schematic of IgG-antibody/ Mannosylation detection by Protein A/Lectin functionalized sensors. Panels A and B adapted from ref 47. Copyright 2011 American Chemical Society. Panel C adapted from ref 46. Copyright 2013 American Chemical Society.

analytes to alter the light emission; highly chirality-enriched SWCNTs are used to make solar cells; ${ }^{44}$ and the thermal conductivity, thermoelectricity, and electrical conductivity of SWCNTs are used to generate electrical energy from chemical energy in thermopower waves. ${ }^{49,50}$

3.2. Single Molecule Detection and Sensors Based on Near-Infrared Photoluminescence from Carbon Nanotubes. The past decade has seen a rapid emergence in the use of nanomaterials for sensing applications including the development of high-sensitivity and high-specificity optical biosensors. When photoexcited by light in the visible range, semiconducting single-walled carbon nanotubes (SWCNTs) absorb the light to form an exciton, which can then radiatively emit in the near-infrared (nIR) range. ${ }^{51}$ Although they are direct band gap semiconductors, the quantum yield (QY) of SWCNTs is typically about $1 \%$ due to a variety of nonradiative decay routes and dark states. The exciton recombination lifetime is on the order of tens to hundreds of picoseconds. ${ }^{52}$ Semiconducting SWCNTs exhibit two essential features required for their use as fluorescence-based sensors of biological activity: (1) SWNTs are the only fluorophores to date that have essentially no photobleaching limit, and (2) they emit light in the nIR, an optical window where tissues, cells, blood, and other biological samples are transparent. ${ }^{53}$ All the carbon atoms in a nanotube are surface atoms, and therefore, small perturbations in the SWCNT environment, known as the "corona", can have large effects on their electronic and optical properties.

SWNTs are often noncovalently functionalized to enable their use as sensors in aqueous environments, which also reduces SWNT toxicity by masking their hydrophobic nature. ${ }^{54}$
However, carefully chosen functionalization schemes can also impart sensing capabilities to SWNT. In the presence of a specific analyte molecule, the nIR emission of the SWCNT can change in intensity or wavelength. Structural differences among SWCNTs modulate the photoluminesent properties. Individual SWCNTs are defined by their chiral vector or the direction of rolling along the hexagonal graphene lattice. Each chirality has a specific set of excitation and emission wavelengths corresponding to energy levels in the electronic structure of the SWCNT, so that SWCNTs of different chiralities can serve as different fluorophore "colors" for multiplexed sensor responses. ${ }^{55}$ Recent advances have enabled high-throughput separation of different SWCNT chiralities, which opens the door for more controlled sensor design via ratiometric sensing. ${ }^{56}$ For an extensive overview on fluorescence-based carbon nanotube sensors, we refer the reader to the literature. ${ }^{57}$

Several characteristics determine the relative effectiveness of SWCNT-based optical sensors. First, a sensor must be selective for a specific analyte, in particular among other potential interfering molecules that are likely to be present in the environment where the sensor will be employed. Second, a sensor must be sensitive for an appreciable analyte concentration range, where single-molecule detection is the ideal detection limit. Third, a sensor signal should show no hysteresis. These features have been achieved in our group in SWCNT-based $\mathrm{H}_{2} \mathrm{O}_{2}$ and $\mathrm{NO}$ sensors. ${ }^{47,58} \mathrm{H}_{2} \mathrm{O}_{2}$ and $\mathrm{NO}$ serve as important messengers in cellular signaling, but they are small reactive molecules with short lifetimes that are difficult to measure. The SWCNT-based sensors were rendered specific to $\mathrm{H}_{2} \mathrm{O}_{2}$ or $\mathrm{NO}$ by wrapping them in different polymers. When these reactive species adsorb onto the surface of specifically 
A

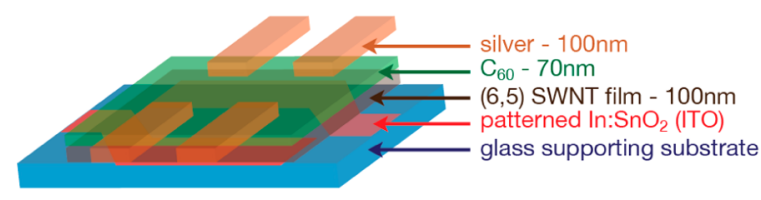

C

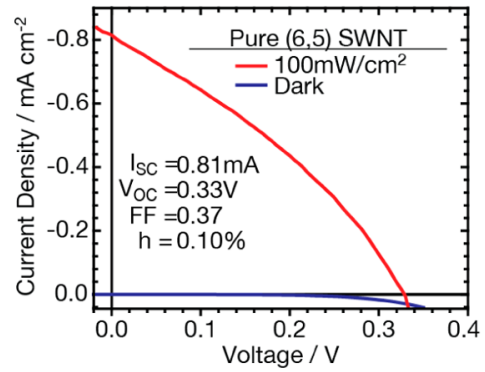

B

D
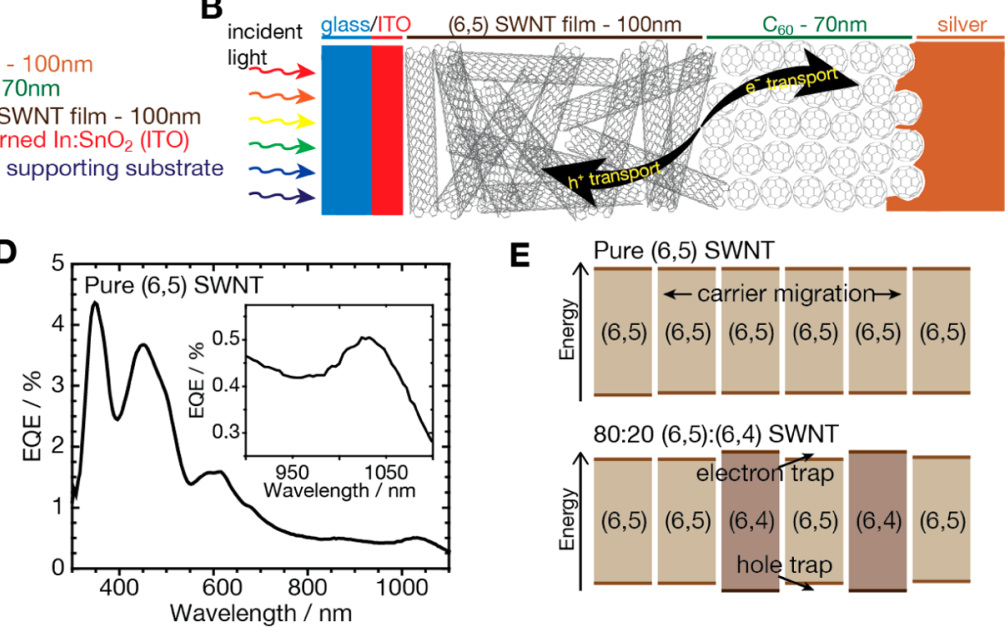

$\mathbf{E}$

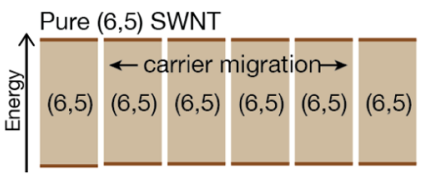

80:20 (6,5):(6,4) SWNT

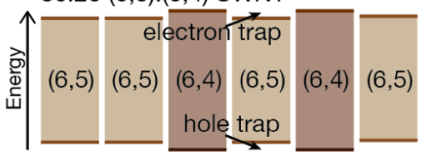

Figure 6. Schematic of $(6,5)$ single walled carbon nanotube-based photovoltaic produced in ref 44 (A). Side schematic of the photovoltaic showing the planar interface for exciton dissociation and expected random SWNT morphology (B). (C) Current-voltage curve and (D) external quantum efficiency (EQE) spectrum of a typical device showing an overall efficiency of $0.1 \%$ and an EQE peak at the absorption peak of the SWNT film in the near-infrared. Energy traps are likely cause by the mixture of different chirality SWNTs that prevent exciton mobility across heterogeneous mixtures of tubes (E). All panels adapted with permission from ref 44. Copyright 2012 Wiley.

functionalized SWCNT sensors, they quench SWCNT fluorescence. Importantly, no hysteresis is observed: when the reactive molecules desorb, fluorescence is recovered. Singlemolecule adsorption/desorption events can also be observed at low concentrations of $\mathrm{H}_{2} \mathrm{O}_{2}$ and $\mathrm{NO}$, as evidenced by single steps in the nIR intensity-time traces of individual SWCNTs (see Figure 5A,B). Interestingly, these sensors were successful in detecting $\mathrm{H}_{2} \mathrm{O}_{2}$ efflux and NO production in cells.

An important approach to the sensitization of SWCNTs toward specific molecules is to directly couple biological recognition elements such as antibodies to the nanotube surface. For SWCNT sensors, this approach necessitates that a recognition element be immobilized within the "corona" of the nanotube. To achieve this design, we suspended SWCNTs in chitosan and functionalized them with nitrilotriacetic acid (NTA) groups, which coordinate $\mathrm{Ni}$ ions. This approach allowed the site-directed immobilization of diverse His-tagged proteins and was used to study protein-protein interactions. ${ }^{59}$ The NTA/His-tag functionalization scheme is generic and can be extended to practically any His-tagged biomolecule (see Figure 5C). This approach was recently used to detect troponin, a biomarker for heart attacks. ${ }^{48}$

These results exemplify the detection of well-defined analytes. However, in nature, biomolecules are often not monodisperse, as in the different glycosylation patterns of antibodies. Toward this end, SWCNT-based sensors were used to detect antibodies (IgG) and determine affinity distributions. ${ }^{46}$ To accomplish affinity distribution detection, SWCNT wrapped with chitosan were functionalized with Protein A, which binds the Fc-domain of antibodies (see Figure 5C). These sensors were subsequently incorporated in a polyacrylamide gel to prevent non-specific protein adsorption. With this platform, affinity distributions of different IgG samples and even production rates of IgG-secreting cells were measured.

Outlook. Recent results in SWCNT-based optical sensing suggest many promising directions for developing future sensors. The rational design of SWCNT-based sensors will undoubtedly hinge on our ability to exploit molecular recognition within the SWCNT corona. While using known molecular recognition units such as antibodies is standard for achieving specificity, doing so bears several disadvantages. Primarily, naturally occurring antibodies do not always exist for a particular analyte, so the rational design of synthetic antibodies will be required. Moreover, all intrinsic disadvantages such as cross-reactivity or low affinity are transferred to any sensor system that relies on these moieties to provide molecular recognition. Future directions in the design of SWCNT-based sensors will require scientists to design target recognition elements within the SWCNT corona for the development of novel sensors.

3.3. All-Carbon Photovoltaic. Recent advances in the separation of highly purified single-chirality single-walled carbon nanotubes (SWCNTs) $)^{56,60,61}$ has enabled a new class of optoelectronic devices ${ }^{62-64}$ and furthered the understanding of chirality dependence on the performance of polymercarbon nanotube heterojunction devices. ${ }^{65-67}$ Bindl et al. demonstrated for the first time the use of SWCNTs as the active absorber in both planar ${ }^{62}$ and bulk ${ }^{63}$ heterojunction photovoltaic configurations. This initial demonstration proved the potential of SWCNTs as near-infrared (nIR) absorbers to efficiently harness energy in the 1000 to $1400 \mathrm{~nm}$ range. ${ }^{62,63,68}$ Since then, several SWCNT-enabled photovoltaics (PVs) have been developed, most of which have required the use of polymers, which served as agents to wrap and surround SWCNTs ${ }^{62,63,69,70}$ or as direct components of the photoactive layer. ${ }^{65-67}$ While such layers are expected to increase device performance at the laboratory scale, the use of PV engineered polymers is often restrictive due to low photostability and the necessity of highly controlled environments for device assembly and characterization. ${ }^{71}$ In contrast, carbon nanotubes have the strong advantage of being extremely stable in air while at the same time absorbing in the near-infrared region of the solar spectrum. $^{72}$

Our group demonstrated for the first time a polymer-free carbon-based photovoltaic which relies on exciton dissociation at a planar SWCNT/ $\mathrm{C}_{60}$ heterojunction interface (Figure $6 \mathrm{~A}, \mathrm{~B}) .{ }^{44}$ We showed that single chirality $(6,5)$ semiconducting SWCNT films are able to generate excitons that can be dissociated at a $\mathrm{C}_{60}$ interface with $0.5 \%$ external quantum efficiency (EQE) at the peak of the nanotube absorption 
A
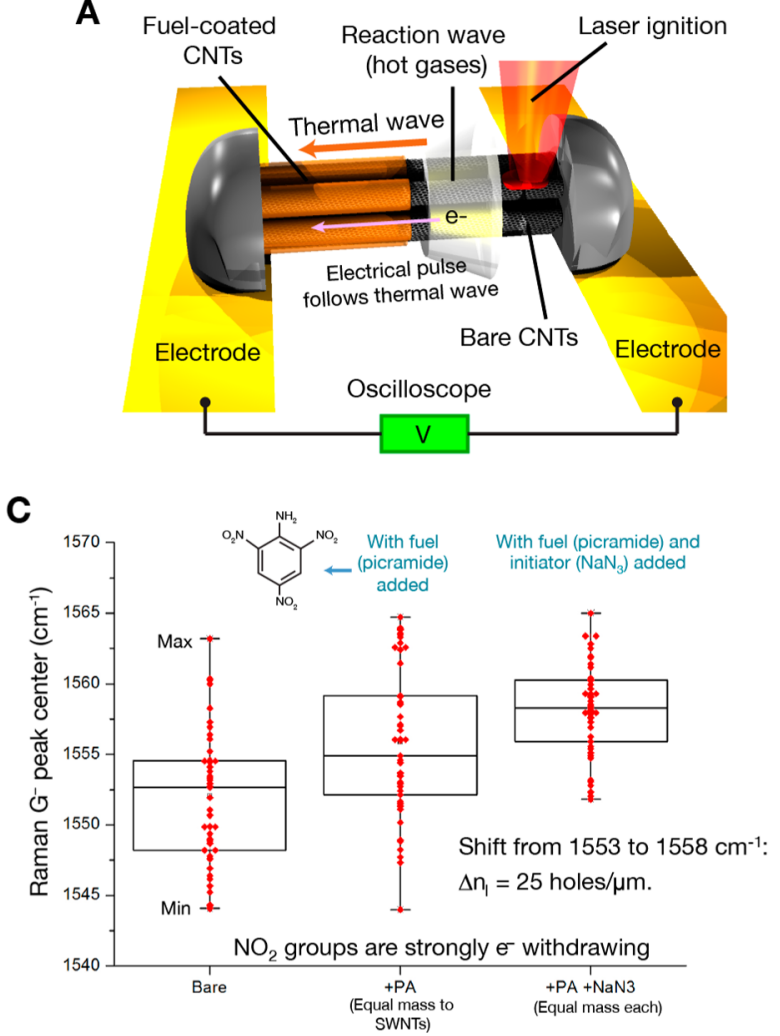

B
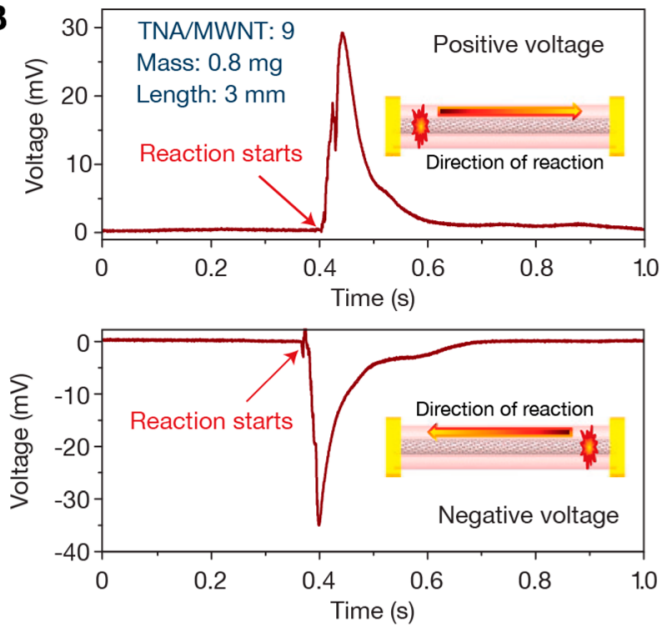

D

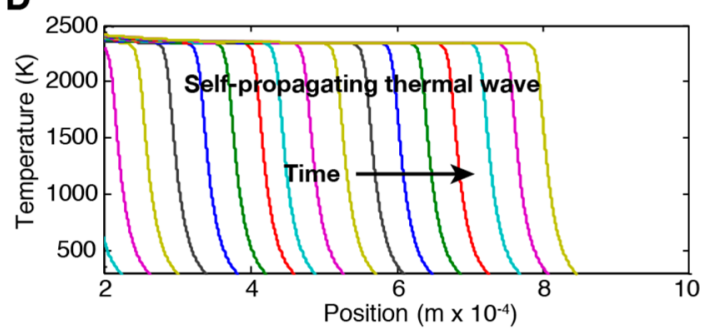

Figure 7. Thermopower wave devices and analysis. (A) Schematic of operation of a thermopower wave generator. ${ }^{50}$ (B) Examples of voltage output curves of positive and negative polarity corresponding to opposite wave propagation directions. The voltage rises as the wave propagates and decreases when the temperature gradient dissipates. ${ }^{49}$ (C) Shift of $\mathrm{G}^{-}$peak position in Raman spectra of nanotubes when bare, when coated with fuel, and when coated with fuel and initiator, showing degree of hole doping. ${ }^{50}$ (D) Temperature waves from numerical simulations showing selfpropagating waves at different times. ${ }^{91}$ Panels A and C adapted from ref 50. Copyright 2013 American Chemical Society. Panel B adapted with permission from ref 49. Copyright 2010 Nature Publishing Group. Panel D adapted from ref 91. Copyright 2010 American Chemical Society.

spectrum (Figure 6C,D). The overall device efficiency under AM1.5 illumination is $0.1 \%$ efficient (Figure 6C). However, when the active layer contained $20 \%(6,4)$ SWCNT, the efficiency was reduced by over 40 -fold. We were hence able to show that heterogeneous mixtures of SWNT limit charge extraction, likely due to the formation of traps in the path of travel of the charge (Figure 6E). Further, we showed that the SWCNT layer and the $\mathrm{C}_{60}$ layer do not adhere strongly to each other, potentially yielding poor charge separation at the interface. ${ }^{44}$ Bernardi et al. also developed an all-carbon PV, this time in a bulk heterojunction configuration with PCBM as the exciton generating layer and reduced graphene oxide (rGO) driving type II dissociation and hole transfer to SWCNTs. ${ }^{41}$ They achieved $1.3 \%$ EQE with a mixture of semiconducting SWCNTs.

This work has led to additional devices with a variety of chemical and morphological configurations. ${ }^{73-75}$ Progress is also being made in more refined and scalable control of morphology ${ }^{42,76}$ and improved understanding of optical response ${ }^{77,78}$ and exciton ${ }^{79,80}$ and charge transport. ${ }^{81}$ Morphology is expected to play a substantial role in solar cell performance. It has been shown that when the SWNT network is not vertically aligned, exciton diffusion is dominated by exciton energy transfer (EET) between nanotubes, limiting the diffusion length in the current-axis to $<10 \mathrm{~nm}$, versus $>100 \mathrm{~nm}$ diffusion lengths observed along the SWCNT axis. ${ }^{74}$ From a charge transport perspective, this observation suggests that a vertically aligned film may be an optimal geometry to minimize exciton trapping in films of mixed nanotube chiralities, allowing for systems composed of a variety of nanotube chiralities to absorb a wide range of wavelengths. This consideration is confounded, however, by the depolarization effect, which attenuates electric fields polarized perpendicular to the SWCNT long axis, reducing the total UV-vis-nIR (ultravioletvisible-near-infrared) absorption cross-section by a factor of $\sim 5$ from a horizontally to vertically aligned film. ${ }^{82}$ An optimal angle is therefore expected. ${ }^{83}$ Recent theoretical evaluation of this phenomenon has been made via kinetic Monte Carlo simulations. $^{84}$

Relative to other organic PVs, the stability of SWCNT-based materials and the low cost potential derived from solution processability and limited input materials make further progress in SWCNT PVs attractive. With the advances made in the separation of single chirality SWCNT, control over solid-state geometry, and understanding of physical phenomena underlying operation, the applications of homogeneous electronic type SWCNT to photovoltaics will enable the next generation of highly efficient nIR PVs.

3.4. Energy Generation, Conversion, and Transport in Thermopower Waves. The unique electrical, thermal, and mechanical properties of low dimensional materials make them ideal candidates for energy generation and transport technologies. In particular, carbon nanotubes (CNTs) have high thermal conductivity of about $3000(\mathrm{~W} / \mathrm{m} / \mathrm{K}) .{ }^{85}$ In our lab, we have developed thermopower waves as a new concept in energy generation and conversion, where a highly exothermic 
chemical reaction is coupled to $1 \mathrm{D}$ conduits with high thermal conductivity to convert chemical energy to electrical energy. ${ }^{49}$ In a typical thermopower wave device, a chemical fuel layer is coated around a thermally and electrically conductive core whose ends are connected to an oscilloscope, as shown in Figure $7 \mathrm{~A}$. The thermal reaction is initiated by an external source such as a short laser pulse. The heat from the exothermic reaction of the fuel is transported forward along the length of the conduit, which in turn causes reaction of the fuel that lies in its path. Thus, a self-propagating reaction wave is launched, and a voltage output $V_{\text {out }}$ is obtained across the ends of the thermal conduit.

Thermopower waves were first launched using multiwalled carbon nanotubes (MWCNTs) as the conduit, coated in cyclotrimethylene trinitramine (TNA) as the fuel and sodium azide added as an initiator to lower the energy of activation. ${ }^{49}$ The measured power density was as high as $7 \mathrm{~kW} / \mathrm{kg}$, and the output voltage was up to $210 \mathrm{mV}^{49}$ As shown in Figure $7 \mathrm{~B}$, the voltage pulse arises when the reaction starts and decreases as the temperature drops, with the polarity depending on the direction of the wave propagation.

To explain the voltage generated by thermopower waves, we consider that carbon nanotubes show a Seebeck coefficient of about $80 \mu \mathrm{V} / \mathrm{K}^{86}$ The temperature difference between the reacting wavefront and the cold (unreacted) side of the conduit is typically on the order of $1000 \mathrm{~K}$, so the Seebeck effect contributes to output voltage by conventional thermoelectric effect $\left(V_{\mathrm{TE}}\right)$. However, the output voltage is much higher than that predicted by thermoelectricity. This "excess voltage" was found to occur because of the phenomena of "excess thermopower" $P_{\mathrm{xs}}$ arising out of the changing chemical potential gradient as the nanotubes are reacted: ${ }^{50}$

$$
P_{\mathrm{xs}}=\frac{V_{\mathrm{out}}{ }^{2}}{R_{\mathrm{c}}}-\frac{V_{\mathrm{TE}}{ }^{2}}{R_{\mathrm{c}}}
$$

where $R_{\mathrm{c}}$ is the circuit load and $V_{\text {out }}$ is the measured output voltage. The adsorption of fuel molecules, which contain many electron-withdrawing $\mathrm{NO}_{2}$ groups, on carbon nanotubes dopes them and causes a change in the chemical potential. As this fuel reacts, the chemical potential gradient between the reacted and unreacted sides (or the undoped and doped sides) leads to an additional electrical output we call "excess thermopower". Experimental evidence of the doping of fuels is shown in Figure $7 \mathrm{C}$, where the $\mathrm{G}^{-}$Raman peak of CNTs upshifts. ${ }^{50}$ The theory of excess thermopower explains superior performance of thermopower wave generators over conventional thermoelectrics and opens up avenues for improving efficiency of operation by controlled doping of CNTs. ${ }^{50,87}$

A variety of different conduits and fuels have been pursued for thermopower waves. Walia et al. have performed experiments with bismuth telluride $\left(\mathrm{Bi}_{2} \mathrm{Te}_{3}\right)$ deposited on alumina and terracotta with nitrocellulose as the fuel. ${ }^{86}$ The high Seebeck coefficient of $\mathrm{Bi}_{2} \mathrm{Te}_{3}$ (about $287 \mu \mathrm{V} / \mathrm{K}$ ) used in conjunction with high thermal conductivity alumina gave electrical power output as high as $10 \mathrm{~mW}$, with maximum voltage up to $150 \mathrm{mV}$. Their analysis also showed lack of oscillations in output when using $\mathrm{Bi}_{2} \mathrm{Te}_{3}$ with terracotta, a comparatively low thermal conductivity material. ${ }^{86}$ Other works in this field explore using other conduit materials such as $\mathrm{Sb}_{2} \mathrm{Te}_{3}{ }^{88} \mathrm{MnO}_{2}{ }^{89}$ and $\mathrm{ZnO} .^{90}$

Analytical work in this area has also advanced the understanding of self-propagating reaction waves. The inverse adiabatic reaction temperature is given by $\beta=\left(C_{\mathrm{p}} E_{\mathrm{a}}\right) /((-\Delta H)$ $R)$ where $C_{\mathrm{p}}$ is the specific heat, $E_{\mathrm{a}}$ is the energy of activation of the reaction, $(-\Delta H)$ is the heat of reaction, and $R$ is the specific gas constant. ${ }^{91,92}$ We used numerical simulations of the governing heat and mass balance equations to show that, as the thermopower wave progresses, the temperature behind the reaction wavefront is equal to $(1 / \beta)$. An expression governing the constant velocity of propagation of such waves was found by using a logistic form of the equation to describe the temperature profile of a self-propagating reaction wave. ${ }^{91}$ The self-propagating thermal waves from numerical simulation are shown in Figure 7D. Numerical analysis also showed that, by appropriately tuning the properties of the fuel and the thermal conduit, it is possible to obtain a self-propagating wave with an oscillating velocity and voltage output. As predicted by simulations, voltage oscillations in the range of $0.4-5 \mathrm{kHz}$ were experimentally demonstrated using a system of TNA on MWCNTs. $^{93}$

\section{CONCLUSIONS AND OUTLOOK}

Low dimensional materials control the transport of molecules by physical constraints that are similar in size. Of the two systems described in this work, the transport through carbon nanotubes has received the most attention to date. However, our work illustrates the shortfall between theory, simulation, and experiment. Because the structure of water can vary so abruptly between the 0.9 and $2.0 \mathrm{~nm}$ range due to its altered phase behavior and physical constraints, essentially distinct treatments are needed for each chiral species in this set. Graphene membranes and their transport behavior are still in their infancy, with many questions remaining regarding how to control the structure and chemistry of their pores. This early progress in graphene and nanotube nanopores suggests promise for applications in membrane and barrier technologies. Near infrared fluorescent, semiconducting carbon nanotubes have proven to be effective sensors that respond to single molecules of analyte, thereby achieving the ultimate sensitivity limit for any platform. However, the challenge remains to design and fabricate interfaces that are selective to one particular molecule over another. Low dimensional materials also comprise some of the best conductors of thermal energy and photogenerated excitons due to the physical confinement of these quasiparticles. Their application to energy technologies that specifically exploit these properties is just beginning, however. The all-carbon photovoltaic offers an alternative to polymer or inorganic photovoltaic devices or photoelectrochemical cells in comprising the junction between two nanocarbon interfaces with increased order for carrier and exciton transport. Thermopower waves and other wave phenomena can be used for energy generation only in specific nanoconduits and remain untapped for storage, transportation, and power applications. These energy transport applications are still in an early stage of development, but commercial applications of carbon nanomaterials in polymer composites suggest promise for further development of all-carbon energy applications. As future efforts expand the number of low dimensional materials available for exploration, many opportunities will exist to further study material and energy transport in these exotic systems.

\section{AUTHOR INFORMATION}

Notes

The authors declare no competing financial interest. 


\section{Biographies}

Qing Hua Wang is a postdoctoral research associate in chemical engineering at the Massachusetts Institute of Technology (MIT). She received her B.A.Sc. in Engineering Science at the University of Toronto and her Ph.D. in Materials Science and Engineering at Northwestern University. Her research interests include self-assembly, chemistry of $2 \mathrm{D}$ nanomaterials, and scanning probe microscopy.

Michael S. Strano is Professor of Chemical Engineering at the Massachusetts Institute of Technology (MIT). His research focuses on biomolecule/nanoparticle interactions and the surface chemistry of low-dimensional systems, nanoelectronics, nanoparticle separations, and applications of vibrational spectroscopy to nanotechnology. Michael has received numerous awards for his work and was ranked among the top 20 chemists of the past decade by Thomson Reuters based on citation impact scores for chemistry publications (http:// web.mit.edu/stranogroup/).

\section{REFERENCES}

(1) Holt, J. K.; Park, H. G.; Wang, Y.; Stadermann, M.; Artyukhin, A. B.; Grigoropoulos, C. P.; Noy, A.; Bakajin, O. Science 2006, 312, 1034.

(2) Garaj, S.; Hubbard, W.; Reina, A.; Kong, J.; Branton, D.; Golovchenko, J. A. Nature 2010, 467, 190.

(3) Noy, A.; Park, H. G.; Fornasiero, F.; Holt, J. K.; Grigoropoulos, C. P.; Bakajin, O. Nano Today 2007, 2, 22.

(4) Koenig, S. P.; Wang, L.; Pellegrino, J.; Bunch, J. S. Nat. Nanotechnol. 2012, 7, 728.

(5) Lee, C. Y.; Choi, W.; Han, J.-H.; Strano, M. S. Science 2010, 329, 1320.

(6) Choi, W.; Lee, C. Y.; Ham, M.-H.; Shimizu, S.; Strano, M. S. J. Am. Chem. Soc. 2010, 133, 203.

(7) Takaiwa, D.; Hatano, I.; Koga, K.; Tanaka, H. Proc. Natl. Acad. Sci. 2008, 105, 39.

(8) Majumder, M.; Chopra, N.; Andrews, R.; Hinds, B. J. Nature 2005, 438, 44.

(9) Fornasiero, F.; Park, H. G.; Holt, J. K.; Stadermann, M.; Grigoropoulos, C. P.; Noy, A.; Bakajin, O. Proc. Natl. Acad. Sci. 2008, 105,17250 .

(10) Majumder, M.; Chopra, N.; Hinds, B. J. J. Am. Chem. Soc. 2005, 127, 9062.

(11) Choi, W.; Ulissi, Z. W.; Shimizu, S. F. E.; Bellisario, D. O.; Ellison, M. K.; Strano, M. S. Nat. Commun. 2013, 4, 2397.

(12) Drahushuk, L. W.; Strano, M. S. Langmuir 2012, 28, 16671.

(13) Cao, D.; Pang, P.; He, J.; Luo, T.; Park, J. H.; Krstic, P.; Nuckolls, C.; Tang, J.; Lindsay, S. ACS Nano 2011, 5, 3113.

(14) Pang, P.; He, J.; Park, J. H.; Krstic, P. S.; Lindsay, S. ACS Nano 2011, 5, 7277.

(15) Thomas, J. A.; McGaughey, A. J. Nano Lett. 2008, 8, 2788.

(16) Xue, Q.; Jing, N.; Chu, L.; Ling, C.; Zhang, H. RSC Adv. 2012, 2, 6913.

(17) Nelson, P. H. AAPG Bull. 2009, 93, 329.

(18) Maniwa, Y.; Kataura, H.; Abe, M.; Udaka, A.; Suzuki, S.; Achiba, Y.; Kira, H.; Matsuda, K.; Kadowaki, H.; Okabe, Y. Chem. Phys. Lett. 2005, 401, 534.

(19) Maniwa, Y.; Kataura, H.; Abe, M.; Suzuki, S.; Achiba, Y.; Kira, H.; Matsuda, K. J. Phys. Soc. Jpn. 2002, 71, 2863.

(20) Webber, J. B. W. Prog. Nucl. Magn. Reson. Spectrosc. 2010, 56, 78. (21) Chen, Q.; Herberg, J. L.; Mogilevsky, G.; Wang, H.-J.; Stadermann, M.; Holt, J. K.; Wu, Y. Nano Lett. 2008, 8, 1902.

(22) Erko, M.; Findenegg, G. H.; Cade, N.; Michette, A. G.; Paris, O. Phys. Rev. B 2011, 84, 104205.

(23) Jahnert, S.; Vaca Chavez, F.; Schaumann, G. E.; Schreiber, A.; Schonhoff, M.; Findenegg, G. H. Phys. Chem. Chem. Phys. 2008, 10, 6039.

(24) Radhakrishnan, R.; Gubbins, K. E.; Sliwinska-Bartkowiak, M. J. Chem. Phys. 2000, 112, 11048.

(25) Kanda, H.; Miyahara, M. J. Chem. Phys. 2007, 126, 054703.
(26) Miyahara, M.; Kanda, H.; Shibao, M.; Higashitani, K. J. Chem. Phys. 2000, 112, 9909.

(27) Miyahara, M.; Kanda, H.; Yoshioka, T.; Okazaki, M. Langmuir 2000, 16, 4293.

(28) Alexiadis, A.; Kassinos, S. Chem. Rev. 2008, 108, 5014.

(29) Frenkel, D.; Smit, B. Understanding Molecular Simulation: From Algorithms to Applications (Computational Science), Second ed.; Academic Press: New York, 2001.

(30) Miyahara, M.; Gubbins, K. E. J. Chem. Phys. 1997, 106, 2865.

(31) Rasaiah, J. C.; Garde, S.; Hummer, G. Annu. Rev. Phys. Chem. 2008, 59, 713.

(32) Peterson, K.; Feller, D.; Dixon, D. Theor. Chem. Acc. 2012, 131, 1.

(33) Arab, M.; Picaud, F.; Devel, M.; Ramseyer, C.; Girardet, C. Phys. Rev. B 2004, 69, 165401.

(34) Moulin, F.; Devel, M.; Picaud, S. Phys. Rev. B 2005, 71, 165401.

(35) Bunch, J. S.; Verbridge, S. S.; Alden, J. S.; van der Zande, A. M.; Parpia, J. M.; Craighead, H. G.; McEuen, P. L. Nano Lett. 2008, 8, 2458.

(36) Jiang, D.-e.; Cooper, V. R.; Dai, S. Nano Lett. 2009, 9, 4019.

(37) Blankenburg, S.; Bieri, M.; Fasel, R.; Müllen, K.; Pignedoli, C. A.; Passerone, D. Small 2010, 6, 2266.

(38) O’Hern, S. C.; Stewart, C. A.; Boutilier, M. S. H.; Idrobo, J.-C.; Bhaviripudi, S.; Das, S. K.; Kong, J.; Laoui, T.; Atieh, M.; Karnik, R. ACS Nano 2012, 6, 10130.

(39) Du, H.; Li, J.; Zhang, J.; Su, G.; Li, X.; Zhao, Y. J. Phys. Chem. C 2011, 115, 23261.

(40) Schrier, J. ACS Appl. Mater. Interfaces 2012, 4, 3745.

(41) Bernardi, M.; Lohrman, J.; Kumar, P. V.; Kirkeminde, A.; Ferralis, N.; Grossman, J. C.; Ren, S. Q. ACS Nano 2012, 6, 8896.

(42) Sun, X.; Chen, T.; Yang, Z.; Peng, H. Acc. Chem. Res. 2012, 46, 539.

(43) Berciaud, S.; Cognet, L.; Lounis, B. Phys. Rev. Lett. 2008, 101, 077402.

(44) Jain, R. M.; Howden, R.; Tvrdy, K.; Shimizu, S.; Hilmer, A. J.; McNicholas, T. P.; Gleason, K. K.; Strano, M. S. Adv. Mater. 2012, 24, 4436.

(45) Pop, E.; Mann, D.; Wang, Q.; Goodson, K.; Dai, H. Nano Lett. 2005, 6, 96.

(46) Reuel, N. F.; Grassbaugh, B.; Kruss, S.; Mundy, J. Z.; Opel, C.; Ogunniyi, A.; Egodage, K.; Wahl, R.; Helk, B.; Zhang, J.; Kalcioglu, Z. I.; Tvrdy, K.; Bellisario, D. O.; Mu, B.; Blake, S. S.; Van Vliet, K. J.; Love, J. C.; Wittrup, K. D.; Strano, M. S. ACS Nano 2013, 7, 74727482 .

(47) Zhang, J.; Boghossian, A. A.; Barone, P. W.; Rwei, A.; Kim, J. H.; Lin, D.; Heller, D. A.; Hilmer, A. J.; Nair, N.; Reuel, N. F.; Strano, M. S. J. Am. Chem. Soc. 2011, 133, 567.

(48) Zhang, J.; Kruss, S.; Hilmer, A. J.; Shimizu, S.; Schmois, Z.; Cruz, F. D. L.; Barone, P. W.; Reuel, N. F.; Heller, D. A.; Strano, M. S. Adv. Healthcare Mater. 2013, DOI: 10.1002/adhm.201300033.

(49) Choi, W.; Hong, S.; Abrahamson, J. T.; Han, J.-H.; Song, C.; Nair, N.; Baik, S.; Strano, M. S. Nat. Mater. 2010, 9, 423.

(50) Abrahamson, J. T.; Sempere, B.; Walsh, M. P.; Forman, J. M.; Şen, F.; Şen, S.; Mahajan, S. G.; Paulus, G. L. C.; Wang, Q. H.; Choi, W.; Strano, M. S. ACS Nano 2013, 7, 6533-6544.

(51) O'Connell, M. J.; Bachilo, S. M.; Huffman, C. B.; Moore, V. C.; Strano, M. S.; Haroz, E. H.; Rialon, K. L.; Boul, P. J.; Noon, W. H.; Kittrell, C.; Ma, J. P.; Hauge, R. H.; Weisman, R. B.; Smalley, R. E. Science 2002, 297, 593.

(52) Hagen, A.; Steiner, M.; Raschke, M. B.; Lienau, C.; Hertel, T.; Qian, H.; Meixner, A. J.; Hartschuh, A. Phys. Rev. Lett. 2005, 95, 197401.

(53) Boghossian, A. A.; Zhang, J.; Barone, P. W.; Reuel, N. F.; Kim, J. H.; Heller, D. A.; Ahn, J. H.; Hilmer, A. J.; Rwei, A.; Arkalgud, J. R.; Zhang, C. T.; Strano, M. S. ChemSusChem 2011, 4, 848.

(54) Sayes, C. M.; Liang, F.; Hudson, J. L.; Mendez, J.; Guo, W.; Beach, J. M.; Moore, V. C.; Doyle, C. D.; West, J. L.; Billups, W. E.; Ausman, K. D.; Colvin, V. L. Toxicol. Lett. 2006, 161, 135. 
(55) Heller, D. A.; Jin, H.; Martinez, B. M.; Patel, D.; Miller, B. M.; Yeung, T. K.; Jena, P. V.; Hobartner, C.; Ha, T.; Silverman, S. K.; Strano, M. S. Nat. Nanotechnol. 2009, 4, 114.

(56) Liu, H.; Nishide, D.; Tanaka, T.; Kataura, H. Nat. Commun. 2011, 2, 309.

(57) Kruss, S.; Hilmer, A. J.; Zhang, J.; Reuel, N. F.; Mu, B.; Strano, M. S. Adv. Drug Delivery Rev. 2013, DOI: 10.1016/j.addr.2013.07.015. (58) Jin, H.; Heller, D. A.; Kalbacova, M.; Kim, J. H.; Zhang, J.; Boghossian, A. A.; Maheshri, N.; Strano, M. S. Nat. Nanotechnol. 2010, 5,302 .

(59) Ahn, J. H.; Kim, J. H.; Reuel, N. F.; Barone, P. W.; Boghossian, A. A.; Zhang, J.; Yoon, H.; Chang, A. C.; Hilmer, A. J.; Strano, M. S. Nano Lett. 2011, 11, 2743.

(60) Arnold, M. S.; Green, A. A.; Hulvat, J. F.; Stupp, S. I.; Hersam, M. C. Nat. Nanotechnol. 2006, 1, 60.

(61) Ghosh, S.; Bachilo, S. M.; Weisman, R. B. Nat. Nanotechnol. 2010, 5, 443.

(62) Bindl, D. J.; Wu, M.-Y.; Prehn, F. C.; Arnold, M. S. Nano Lett. 2010, 11, 455 .

(63) Bindl, D.; Brewer, A.; Arnold, M. Nano Res. 2011, 4, 1174.

(64) Dang, X.; Yi, H.; Ham, M.-H.; Qi, J.; Yun, D. S.; Ladewski, R.; Strano, M. S.; Hammond, P. T.; Belcher, A. M. Nat. Nanotechnol. 2011, 6, 377.

(65) Ham, M.-H.; Paulus, G. L. C.; Lee, C. Y.; Song, C.; Kalantarzadeh, K.; Choi, W.; Han, J.-H.; Strano, M. S. ACS Nano 2010, 4, 6251.

(66) Ren, S. Q.; Bernardi, M.; Lunt, R. R.; Bulovic, V.; Grossman, J. C.; Gradečak, S. Nano Lett. 2011, 11, 5316.

(67) Kanai, Y.; Grossman, J. C. Nano Lett. 2008, 8, 908.

(68) Bindl, D. J.; Safron, N. S.; Arnold, M. S. ACS Nano 2010, 4, 5657.

(69) Arnold, M. S.; Zimmerman, J. D.; Renshaw, C. K.; Xu, X.; Lunt, R. R.; Austin, C. M.; Forrest, S. R. Nano Lett. 2009, 9, 3354.

(70) Ramuz, M. P.; Vosgueritchian, M.; Wei, P.; Wang, C.; Gao, Y.; Wu, Y.; Chen, Y.; Bao, Z. ACS Nano 2012, 6, 10384.

(71) Kippelen, B.; Bredas, J.-L. Energy Environ. Sci. 2009, 2, 251.

(72) Avouris, P.; Freitag, M.; Perebeinos, V. Nature Photon. 2008, 2, 341.

(73) Shea, M. J.; Arnold, M. S. Appl. Phys. Lett. 2013, 102, 243101.

(74) Bindl, D. J.; Shea, M. J.; Arnold, M. S. Chem. Phys. 2013, 413, 29.

(75) Isborn, C. M.; Tang, C.; Martini, A.; Johnson, E. R.; Otero-dela-Roza, A.; Tung, V. C. J. Phys. Chem. Lett. 2013, 4, 2914.

(76) Cao, Q.; Han, S.-j.; Tulevski, G. S.; Zhu, Y.; Lu, D. D.; Haensch, W. Nat. Nanotechnol. 2013, 8, 180.

(77) Barkelid, M.; Steele, G. A.; Zwiller, V. Nano Lett. 2012, 12, 5649.

(78) Cherukuri, T. K.; Tsyboulski, D. A.; Weisman, R. B. ACS Nano 2012, 6, 843.

(79) Crochet, J. J.; Duque, J. G.; Werner, J. H.; Doorn, S. K. Nat. Nanotechnol. 2012, 7, 126.

(80) Ruzicka, B. A.; Wang, R.; Lohrman, J.; Ren, S. Q.; Zhao, H. Phys. Rev. B 2012, 86, 205417.

(81) Gabor, N. M.; Zhong, Z. H.; Bosnick, K.; McEuen, P. L. Phys. Rev. Lett. 2012, 108, 087404.

(82) Reich, S.; Thomsen, C.; Maultzsch, J. Carbon nanotubes: basic concepts and physical properties; Wiley-VCH: Weinheim; Cambridge, 2004.

(83) Arnold, M. S.; Blackburn, J.; Crochet, J.; Doorn, S.; Duque, J.; Mohite, A.; Telg, H. Phys. Chem. Chem. Phys. 2013, 15, 14896-14918.

(84) Wu, M. Y.; Jacobberger, R. M.; Arnold, M. S. J. Appl. Phys. 2013, 113, 204504.

(85) Choi, W.; Abrahamson, J. T.; Strano, J. M.; Strano, M. S. Mater. Today 2010, 13, 22.

(86) Walia, S.; Weber, R.; Latham, K.; Petersen, P.; Abrahamson, J. T.; Strano, M. S.; Kalantar-zadeh, K. Adv. Funct. Mater. 2011, 21, 2072.

(87) Abrahamson, J. T.; Song, C.; Hu, J. H.; Forman, J. M.; Mahajan, S. G.; Nair, N.; Choi, W.; Lee, E.-J.; Strano, M. S. Chem. Mater. 2011, 23, 4557.
(88) Walia, S.; Weber, R.; Sriram, S.; Bhaskaran, M.; Latham, K.; Zhuiykov, S.; Kalantar-zadeh, K. Energy Environ. Sci. 2011, 4, 3558.

(89) Walia, S.; Balendhran, S.; Yi, P.; Yao, D.; Zhuiykov, S.; Pannirselvam, M.; Weber, R.; Strano, M. S.; Bhaskaran, M.; Sriram, S.; Kalantar-zadeh, K. J. Phys. Chem. C 2013, 117, 9137.

(90) Walia, S.; Weber, R.; Balendhran, S.; Yao, D.; Abrahamson, J. T.; Zhuiykov, S.; Bhaskaran, M.; Sriram, S.; Strano, M. S.; Kalantar-zadeh, K. Chem. Commun. 2012, 48, 7462.

(91) Abrahamson, J. T.; Strano, M. S. J. Phys. Chem. Lett. 2010, 1, 3514.

(92) Mahajan, S. G.; Wang, Q. H.; Strano, M. S.; Abrahamson, J. T. AIChE J. 2013, 59, 3333.

(93) Abrahamson, J. T.; Choi, W.; Schonenbach, N. S.; Park, J.; Han, J.-H.; Walsh, M. P.; Kalantar-zadeh, K.; Strano, M. S. ACS Nano 2010, $5,367$. 